\title{
BMJ open Long-term follow-up of cortisol awakening response in patients treated for stress-related exhaustion
}

To cite: Sjörs A, Ljung T, Jonsdottir IH. Long-term follow-up of cortisol awakening response in patients treated for stress-related exhaustion. BMJ Open 2012;2:e001091. doi:10.1136/ bmjopen-2012-001091

- Prepublication history for this paper is available online. To view these files please visit the journal online (http:// dx.doi.org/10.1136/ bmjopen-2012-001091)

Received 29 February 2012 Accepted 18 June 2012

This final article is available for use under the terms of the Creative Commons Attribution Non-Commercial 2.0 Licence; see http://bmjopen.bmj.com

${ }^{1}$ The Institute of Stress Medicine, Gothenburg, Sweden

${ }^{2}$ Rehabilitation Medicine, Department of Medical and Health Sciences, Linköping University, Linköping, Sweden

${ }^{3}$ County Council of Jämtland, R\&D-unit, Östersund,

Sweden

${ }^{4}$ Mid Sweden University, IHV, Östersund, Sweden

${ }^{5}$ Institute of Neuroscience and Physiology, The Sahlgrenska Academy, University of Gothenburg, Gothenburg, Sweden

Correspondence to Dr Anna Sjörs; anna.sjors@vgregion.se

\begin{abstract}
Objectives: Studies on hypothalamus-pituitaryadrenal (HPA) axis activity in stress-related exhaustion and burnout have revealed incongruent results, and few longitudinal studies on clinical populations have been performed. This study was designed to investigate differences in HPA axis activity between patients with stress-related exhaustion and healthy controls and to investigate longitudinal changes in HPA axis activity in the patient group as they entered a multimodal treatment programme.
\end{abstract}

Design: HPA axis activity was assessed through the cortisol awakening response (CAR). Salivary cortisol was sampled at awakening and after $15 \mathrm{~min}$. Follow-up measurements were performed in the patient group after 3, 6, 12 and 18 months.

Setting: An outpatient clinic specialising in stressrelated illness.

Participants: Patients with clinically diagnosed stressrelated exhaustion $(n=162)$ and healthy controls $(\mathrm{n}=79)$.

\section{Primary and secondary outcome}

measures: The primary measure was CAR measured as the difference between the two salivary cortisol samples. Changes in CAR during follow-up were related to changes in symptoms of burnout, depression and anxiety.

Results: Patients showed similar CAR as the controls and their CAR did not change significantly during treatment. No association was found between CAR and symptom development during treatment.

Conclusions: The authors conclude that CAR does not seem to discriminate clinically defined patients with exhaustion from healthy controls and it appears not to change during treatment. CAR, measured as salivary cortisol, at awakening and after $15 \mathrm{~min}$, is thus not a valid marker for stress-related exhaustion.

\section{INTRODUCTION}

Stress-related illness is a major problem in many countries ${ }^{1}$ and presents unique challenges to persons with health problems and for their healthcare providers. Particularly,

\section{ARTICLE SUMMARY}

Article focus

- HPA axis activity measured through CAR in patients with stress-related exhaustion.

- Follow-up assessments of CAR were performed in the patient group during 18 months of treatment.

Key messages

- In this study, CAR did not discriminate patients with exhaustion from healthy controls and CAR did not change during treatment.

- Thus, CAR, measured as salivary cortisol, at awakening and after $15 \mathrm{~min}$, does not seem to be a valid marker for stress-related exhaustion.

Strengths and limitations of this study

- Two major advantages were the longitudinal design with four follow-up assessments and the relatively large sample of patients included in the study.

- A limitation was the single day cortisol sampling at each follow-up assessment and using only two samples per day.

the lack of characteristic clinical signs or diagnostic laboratory abnormalities increases difficulty in assigning a correct diagnosis or treatment. Several partly overlapping concepts, such as burnout, exhaustion disorder (ED), vital exhaustion and chronic fatigue syndrome (CFS), are used in the literature to describe patients with stressrelated exhaustion.

The burnout concept is widely used and internationally recognised as one of several mental health outcomes of chronic stress. ${ }^{2}$ Emotional exhaustion has been put forward as the core element of the burnout syndrome. ${ }^{23}$ However, burnout syndrome is not a clinically defined illness, and a better more selective conceptualisation of stressrelated pathologies is needed. Diagnostic criteria for the medical condition referred to as ED was formulated by the National Board of Health and Welfare in Sweden ${ }^{4}$ to define a more homogenous clinical population regarding the severity of the mental health 
problem and its relation to stress exposure. This study investigates a population of ED patients, but uses the burnout concept in parallel, to facilitate comparisons to related literature. ED and self-reported burnout overlap $88 \%-92 \%$, as previously reported. ${ }^{5}$

It has been hypothesised that the hypothalamuspituitary-adrenal (HPA) axis is involved in the pathogenesis of stress-related illness since it is the central neuroendocrine system involved in long-term adaptation to stress. ${ }^{6}{ }^{7}$ According to Raison and Miller, ${ }^{8}$ a disturbed HPA axis could cause the array of symptoms observed in individuals with stress-related disorders. Most research on associations between stress, HPA axis activity and physical and emotional well-being in humans has relied on correlational data. Much of this research is also crosssectional, showing point-in-time rather than longitudinal associations.

Salivary cortisol awakening response (CAR) is a common method used to assess HPA axis activity. In a recent meta-analysis, Chida and Steptoe ${ }^{9}$ concluded that CAR was negatively related to fatigue, burnout or exhaustion. However, studies on HPA axis regulation specifically in burnout subjects have revealed incongruent results. ${ }^{10}$ In strongly affected individuals on sick leave or with a clinical diagnosis, both elevated ${ }^{11} 12$ and non-elevated ${ }^{13-15}$ awakening cortisol levels, compared with healthy control subjects, have been reported. Studies including subjects still at work but scoring high on burnout also show contradictory results, ranging from signs of a hypoactive HPA axis ${ }^{16}$ to normal HPA axis $^{18}$ or hyperactive axis. ${ }^{19} 20$

These inconsistencies could possibly be explained by the heterogeneity in burnout populations, partly due to differences in diagnostic criteria or use of different burnout scales. Using the term 'burnout' in both clinically diagnosed patients and working populations scoring high on burnout questionnaires makes comparison of results in the literature difficult. Burnout in itself is a process suggested to develop over longer periods of time, implying that the HPA axis may not function the same in persons experiencing early stages of burnout as in persons suffering from more severe and chronic forms of burnout. $^{21}$ Furthermore, relatively small samples have been investigated, especially in studies of clinically diagnosed patients with more severe symptoms, thereby increasing the risk of chance findings in individual studies.

Longitudinal studies of HPA axis activity in stressrelated exhaustion or burnout are scarce. Moch et $a t^{2}$ reported no difference in serum cortisol at baseline but lower levels after 4 months following a stress management intervention in 16 female burnout patients compared with matched controls. Urinary free cortisol levels were consistently lower in the patient group throughout the 4 months. Mommersteeg et $a t^{23}$ measured salivary cortisol in a group of 74 burnout patients before and after treatment and again at follow-up approximately 6 months later. They found no changes in salivary cortisol over time but some indications of a relationship between individual changes in cortisol and changes in burnout symptoms. Thus, it is still unclear whether HPA axis activity changes during the development of and recovery from exhaustion/burnout and whether there is any association with symptom development.

The study was designed to investigate CAR in a relatively large sample of patients with clinically diagnosed stress-related exhaustion compared with healthy controls and to investigate the longitudinal development of CAR during treatment in the patient group. Furthermore, we intended to elucidate potential differences between subgroups of patients based on depression diagnosis, antidepressant use and symptom duration and investigate whether CAR is associated with the development of different symptoms during treatment. Since previous studies have shown contradictory results regarding HPA axis function and burnout/ exhaustion, we considered our study as mainly exploratory. However, given the large sample of patients with such pronounced clinical exhaustion, we hypothesised that a somewhat more hypoactive HPA axis with lower awakening cortisol levels followed by flatter response after awakening would be seen in the patients compared with controls.

\section{MATERIALS AND METHODS \\ Participants}

This study included 162 patients with stress-related exhaustion referred to an outpatient clinic at the Institute of Stress Medicine located in Gothenburg, Sweden. In addition, 85 participants were recruited to serve as healthy controls from an ongoing longitudinal cohort study in the Västra Götaland Region (mainly healthcare workers and social insurance officers) and from advertisements in daily newspapers. The study was approved by the Regional Ethical Review Board in Gothenburg, Sweden, and was conducted in accordance with the Declaration of Helsinki. All participants included in the study gave written informed consent.

The inclusion criteria for patients were (1) clinically diagnosed ED, as defined in box 1 and (2) a maximum duration of sick leave of 6 months. The inclusion criteria for controls were age of 25-50 years and body mass index (BMI) $18.5-30 \mathrm{~kg} / \mathrm{m}^{2}$. None of the controls fulfilled diagnostic criteria for ED.

The exclusion criteria for both patients and healthy controls were as follows: systemic disease (such as thyroid disorder, hypertension or diabetes), psychiatric disease (except for depression, anxiety and exhaustion for the patients), present infection, pregnancy, breastfeeding, medication with substances having systemic effects (except for antidepressants for the patients), vitamin B12 deficiency and over-consumption of alcohol. This was ensured partly by asking participants of known diseases, partly by measuring blood pressure and performing electrocardiographic registration. Blood samples were also taken and blood sedimentation rate and levels of glucose, 
Box 1 Diagnostic criteria for exhaustion disorder as proposed by the Swedish National Board of Health and Welfare

Diagnostic criteria for exhaustion disorder

A. Physical and mental symptoms of exhaustion with minimum 2 weeks duration. The symptoms have developed in response to one or more identifiable stressors that have been present for at least 6 months.

B. Markedly reduced mental energy, which is manifested by reduced initiative, lack of endurance or increased time needed for recovery after mental efforts.

C. At least four of the following symptoms have been present most of the day, nearly every day, during the same 2-week period:

1. Persistent complaints of impaired memory.

2. Markedly reduced capacity to tolerate demands or to work under time pressure.

3. Emotional instability or irritability.

4. Insomnia or hypersomnia.

5. Persistent complaints of physical weakness or fatigue.

6. Physical symptoms such as muscular pain, chest pain, palpitations, gastrointestinal problems, vertigo or increased sensitivity to sounds.

A. The symptoms cause clinically significant distress or impairment in social, occupational or other important areas of functioning.

B. The symptoms are not due to the direct physiological effects of a substance (such as a drug of abuse or a medication) or a general medical condition (eg hypothyroidism, diabetes, infectious disease).

C. If criteria for major depressive disorder, dysthymic disorder or generalised anxiety disorder are met, exhaustion disorder is set as a comorbid condition.

haemoglobin, HbAlc, free T4 and thyroid-stimulating hormone were measured. Psychiatric symptoms were assessed using the Comprehensive Psychopathological Rating Scale and the Hospital Anxiety and Depression (HAD) scale. Vitamin B12 deficiency was assessed by measuring homocysteine, and over-consumption of alcohol was measured with the Alcohol Use Disorders Identification Test. Participant characteristics are shown in table 1 .

\section{Further description of the ED patients}

The patients were ambulatory at the time of study, and none had received inpatient care due to their illness. They were referred from primary care or occupational healthcare centres between 2004 and 2008. Comorbidity of depression and/or anxiety was screened for using the one-page Primary Care Evaluation of Mental Disorders questionnaire, ${ }^{24}$ followed by a structured physician interview using a form based on the DSM IV criteria for diagnostic assessment of mood and anxiety disorders. Patients received individually adapted multimodal treatment and follow-up during 18 months at the clinic as described by Glise et al..$^{25}$ Treatment commonly included advice and discussions of lifestyle and physical
Table 1 Descriptive data of patients and healthy controls

\begin{tabular}{|c|c|c|c|c|}
\hline & $\begin{array}{l}\text { Patients } \\
(n=162)\end{array}$ & $\begin{array}{l}\text { Controls } \\
(n=85)\end{array}$ & $\begin{array}{l}\text { Test } \\
\text { value }\end{array}$ & p Value \\
\hline $\begin{array}{l}\text { Age, mean } \\
\text { (SD) in years }\end{array}$ & $44(9)$ & $45(9)$ & $t=0.5$ & 0.595 \\
\hline Sex (\% male) & $36 \%$ & $51 \%$ & $\chi^{2}=4.6$ & 0.032 \\
\hline $\begin{array}{l}\text { BMI, mean } \\
\text { (SD) in } \mathrm{kg} / \mathrm{m}^{2}\end{array}$ & $24.3(3.0)$ & $23.5(2.4)$ & $\mathrm{t}=-2.2$ & 0.031 \\
\hline $\begin{array}{l}\text { WHR, } \\
\text { mean (SD) }\end{array}$ & $0.87(0.1)$ & $0.87(0.1)$ & $t=1.0$ & 0.324 \\
\hline $\begin{array}{l}\text { Antidepressant } \\
\text { use }\end{array}$ & $27 \%$ & $\mathrm{~N} / \mathrm{A}$ & & \\
\hline $\begin{array}{l}\text { Depression } \\
\text { diagnosis }\end{array}$ & $77 \%$ & $\mathrm{~N} / \mathrm{A}$ & & \\
\hline $\begin{array}{l}\text { Anxiety } \\
\text { diagnosis }\end{array}$ & $75 \%$ & $\mathrm{~N} / \mathrm{A}$ & & \\
\hline $\begin{array}{l}\text { Burnout } \\
\text { score (SMBQ) }\end{array}$ & & & $\chi^{2}=165.8$ & $<0.001$ \\
\hline$<4$ & $8 \%$ & $93 \%$ & & \\
\hline$\geq 4$ & $92 \%$ & $7 \%$ & & \\
\hline $\begin{array}{l}\text { HAD } \\
\text { depression } \\
\text { score }\end{array}$ & & & $\chi^{2}=95.9$ & $<0.001$ \\
\hline $0-6$ & $27 \%$ & $94 \%$ & & \\
\hline $7-10$ & $43 \%$ & $6 \%$ & & \\
\hline$>10$ & $30 \%$ & $0 \%$ & & \\
\hline $\begin{array}{l}\text { HAD anxiety } \\
\text { score }\end{array}$ & & & $\chi^{2}=137.5$ & $<0.001$ \\
\hline $0-6$ & $9 \%$ & $84 \%$ & & \\
\hline $7-10$ & $31 \%$ & $10 \%$ & & \\
\hline$>10$ & $60 \%$ & $6 \%$ & & \\
\hline
\end{tabular}

BMI, body mass index; WHR, waist-hip ratio; SMBQ, ShiromMelamed Burnout Questionnaire; HAD, Hospital Anxiety and Depression Scale.

activity, stress reduction programmes and/or psychological treatment. Antidepressant medication was offered or adjusted when needed.

A total of 274 patients (184 women and 90 men) entered the treatment and follow-up programme at the clinic and among those, 162 patients (103 women and 59 men) provided complete salivary cortisol data from all follow-up assessments and were thus selected for analysis. These 162 patients were significantly older than the 112 excluded patients (mean age for the included patients was 44 years (SD 9) and for the excluded patients was 40 years $(\mathrm{SD} 9), \mathrm{t}=3.9, \mathrm{p}<0.001)$ and had lower burnout scores (median for included patients was 5.3 and median for excluded patients was 5.6, $\mathrm{Z}=2.0, \mathrm{p}=0.048$ ). They did not differ significantly regarding BMI or symptoms of depression and anxiety.

Of the patients selected for analyses, $61 \%$ were diagnosed with both depression and anxiety, $16 \%$ fulfilled requirements for depression only and $15 \%$ for only an anxiety disorder. The self-reported duration of symptoms before seeking medical help was more than 5 years for $11 \%$ of the patients, 3-5 years for $19 \%, 1-2$ years for $29 \%$ and $<1$ year for $42 \%$. Eighty-five per cent of the patients reported some degree of sick leave at inclusion. 


\section{CORTISOL MEASUREMENT PROTOCOL}

To assess individual cortisol response to awakening, saliva samples were collected at home using Salivette tubes (Sarstedt, Nümbrecht, Germany) immediately after waking up and 15 min later. The participants were free to choose a typical weekday for saliva collection, and they were instructed not to brush their teeth, eat or drink anything $30 \mathrm{~min}$ before taking a sample. This instruction was given verbally as well as through detailed written information accompanying the sampling tubes. Collection time was recorded in a paper diary. Female participants were instructed to perform the saliva collection on one day between day 5 and 10 in the menstrual cycle (ie, the follicular phase) since the diurnal cortisol profile could be influenced by the menstrual cycle. ${ }^{26}$ The same procedure was repeated after 3, 6, 12 and 18 months in the patient group.

Salivette tubes were kept refrigerated after collection at home until they were returned to the clinic. The samples were delivered at the next visit, if scheduled within 1 week from sampling or sent by mail. Samples were then stored at $-20^{\circ} \mathrm{C}$ until they were assayed. Free cortisol levels in saliva were analysed by the Laboratory for Clinical Chemistry, Sahlgrenska University Hospital, using a competitive radioimmunoassay (Spectria Coated Tube Radioimmunoassay; Orion Diagnostica, Espoo, Finland). The limit of detection was $1 \mathrm{nmol} / \mathrm{l}$, and the interassay coefficient of variance was below $14 \%$.

\section{QUESTIONNAIRES}

Self-reported burnout was evaluated in this study using the Shirom-Melamed Burnout Questionnaire (SMBQ). The questionnaire includes 22 items measuring different aspects of the burnout syndrome, including physical fatigue, emotional exhaustion, tension, listlessness and cognitive weariness as defined by Melamed and coworkers. ${ }^{27}$ The SMBQ correlates strongly with the Maslach Burnout Inventory, another widely used instrument for measurement of burnout. ${ }^{3}$ Stenlund and coworkers $^{28}$ reported the mean total score in patients with burnout as 5.7 for women and 5.6 for men. In studies of white-collar workers, scores of $\geq 3.75$ on the $\mathrm{SMBQ}$ were used to classify workers with high burnout, and in another study, the median split $\geq 4$ was used as the level for burnout. ${ }^{3}{ }^{12}$ Relating to these previous studies, a score of $\geq 4.0$ on the SMBQ was chosen as the cut-off for burnout in the present study.

The HAD scale was used to assess self-reported depression and anxiety in both patients and controls. It was originally developed for non-psychiatric clinics to measure symptoms of depression (HAD-D) and anxiety (HAD-A), ${ }^{29}$ and the scale has been found to perform well in assessing anxiety disorders and depression in different patient groups as well as in the general population. ${ }^{30}$ Scores $0-6$ indicate non-cases, $7-10$ indicate possible cases and scores above 10 on each respective subscale indicate probable cases.
STATISTICAL ANALYSES

All statistical analyses were conducted in SPSS V.19.0. A p value of $<0.05$ was considered statistically significant. Student's $\mathrm{t}$ tests and $\chi^{2}$ tests were used to compare demographics and questionnaire scores between patients and controls. Cortisol data were analysed with repeated measures analysis of variance (ANOVA). Correction of degrees of freedom according to the Greenhouse-Geisser procedure $(\varepsilon)$ was performed whenever sphericity was violated. Since cortisol data were skewed, all cortisol measurements were ln-transformed before these analyses. Effect sizes were calculated for significant results by partial eta squared $\left(\eta^{2}\right)$, expressing the amount of variance explained in the dependent variable by the respective effect.

\section{CAR in patients and healthy controls}

Salivary cortisol after awakening was analysed using repeated measures ANOVA with sample (awakening and after $15 \mathrm{~min}$ ) as the within-subjects factor and group (patient or control) as the between-subjects factor. The potential confounders age, sex, BMI, waist-hip ratio (WHR), HAD-D and HAD-A were included as covariates in the repeated measures ANOVAs to investigate possible effects on awakening cortisol.

\section{CAR in subgroups of patients}

The difference between the second and first morning sample (15 min after awakening-awakening) was used as a simple measurement of CAR. Relative CAR (relCAR) was computed as CAR/awakening $\times 100$. $t$ Tests were performed to investigate differences in CAR and relCAR between patients scoring $\geq 4$ or $<4$ on the SMBQ and patients with or without depression diagnosis and antidepressant use at inclusion. Moreover, possible differences in CAR and relCAR depending on the level of anxiety and depressive symptoms at inclusion (HAD $<7$, HAD $7-10$ or HAD $>10$ ) were analysed in the patient group by means of one-way ANOVA.

\section{Longitudinal development of CAR}

Longitudinal development was analysed using a repeated measures ANOVA that analysed CAR over the course of treatment. Time (inclusion, 3 months, 6 months, 12 months and 18 months) was applied as the repeated factor, and possible differences between different subgroups of patients were analysed by applying group as a fixed factor and time $\times$ group as an interaction effect. Groupings were made according to depression diagnosis at inclusion (yes or no), antidepressant use (at inclusion, introduced during treatment or no use) and symptom duration before inclusion in the study (more than 5 years, 3-5 years, 1-2 years or $<1$ year), respectively. Age, sex, nicotine use (current, previous or no use), exercise habits (sedentary, light, moderate or vigorous physical activity), BMI and WHR were applied as covariates. 
Associations between CAR and symptom development Possible relationships between CAR and symptom development during treatment were analysed by Spearman rank correlations $(\rho)$ between changes in CAR from inclusion to follow-up at 3, 6, 12 and 18 months and changes in burnout, depressive and anxiety symptoms over the same time intervals.

\section{RESULTS}

\section{CAR in patients and healthy controls}

As burnout and exhaustion are considered closely related, controls with a burnout score $\geq 4(n=6)$ were excluded from all analyses. Both patients and controls showed a significant rise in cortisol after awakening (main effect of sample, $\mathrm{F}(1,238)=174.2, \mathrm{p}<0.001$, $\eta^{2}=0.423$; see figure 1$)$. There was, however, no difference in cortisol level between the patient group and the control group (main effect of group, $\mathrm{F}(1,238)=0.05$, $\mathrm{p}=0.829$ ) or rise in cortisol after awakening (sample $\times$ group interaction effect, $\mathrm{F}(1,238)=0.3, \mathrm{p}=0.615)$. Twenty-one patients (13\%) and 15 controls (19\%) had a negative CAR and excluding these participants did not alter the result. No effect of age, sex, BMI, WHR, HAD-D or HAD-A could be found as they were entered as covariates, either separately or together.

\section{CAR in subgroups of patients}

As seen in figure 2, patients using antidepressants had a slightly smaller rise after awakening, although this difference was not statistically significant in the t tests of CAR $(\mathrm{t}=1.1, \mathrm{p}=0.268)$ and relCAR $(\mathrm{t}=-0.2, \mathrm{p}=0.866)$.

There were no significant differences in CAR or relCAR between patients with or without depression diagnosis at inclusion or between subgroups of patients with various levels of symptoms of burnout, anxiety or depression at inclusion (data not shown).

\section{Longitudinal development of CAR}

Repeated measures ANOVA indicated that CAR did not change significantly over the course of treatment

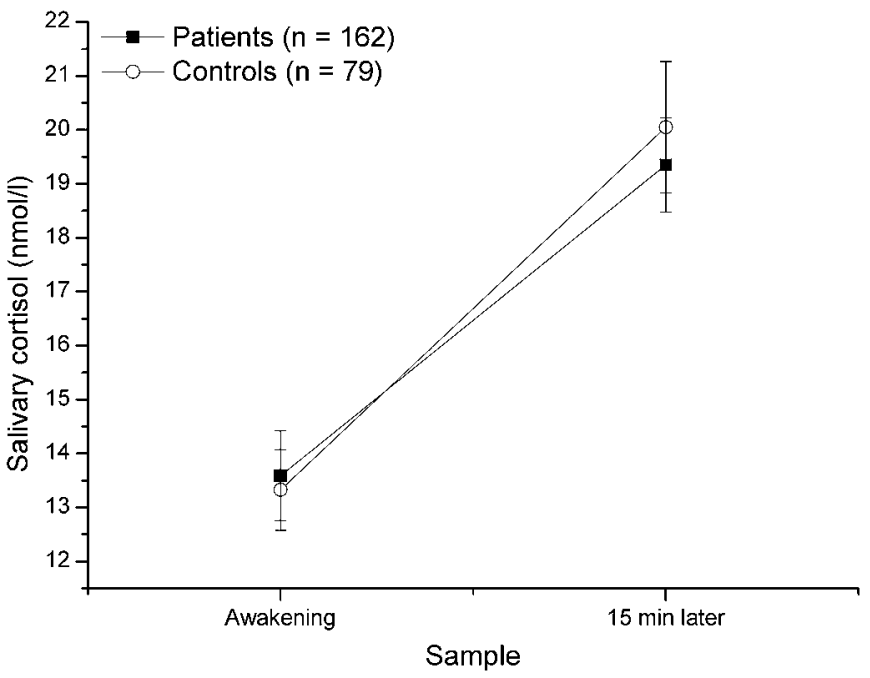

Figure 1 Mean salivary cortisol ( \pm SEM) after awakening in patients with stress-related exhaustion and in healthy controls.

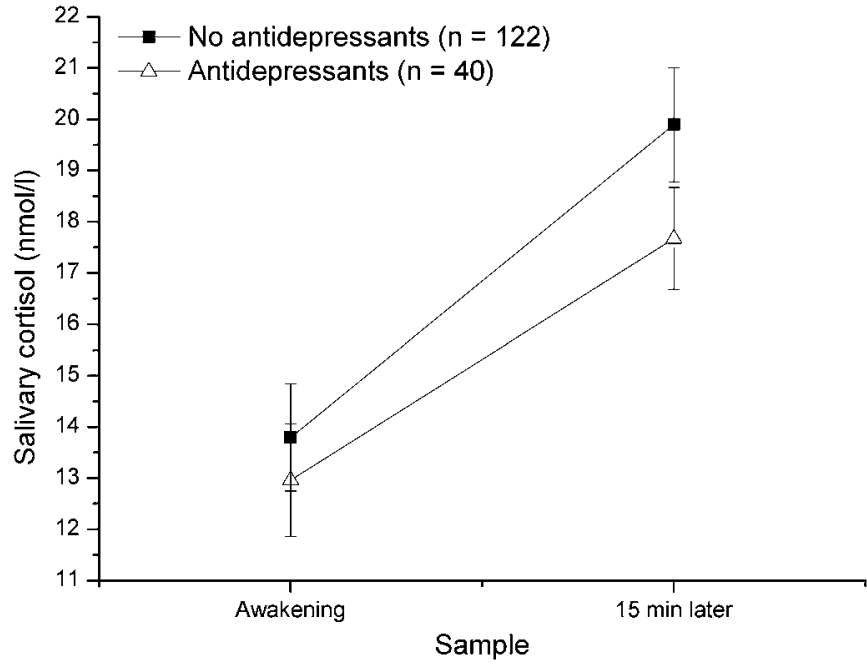

Figure 2 The impact of antidepressant use on mean salivary cortisol ( \pm SEM) after awakening in patients with stress-related exhaustion.

$(\mathrm{F}(4,644)=2.0, \mathrm{p}=0.108, \varepsilon=0.838$, figure 3). Possible impact of antidepressants on CAR in this patient group was investigated further by group division according to antidepressant use. This analysis gave no significant main effect of group $(\mathrm{F}(2,157)=1.4, \mathrm{p}=0.260)$ and no significant time $\times$ group interaction effect $(\mathrm{F}(8,628)=1.2$ $\mathrm{p}=0.304, \varepsilon=0.836$ ), indicating that antidepressant use did not affect CAR during treatment. Likewise, group division according to depression diagnosis at inclusion revealed no significant group $(\mathrm{F}(1,160)=0.1, \mathrm{p}=0.778)$ or interaction effect $(\mathrm{F}(4,640)=0.8, \mathrm{p}=0.501, \varepsilon=0.838)$. There were no significant differences between patients reporting different durations of symptoms before inclusion (group effect, $\mathrm{F}(3,154)=0.8, \mathrm{p}=0.503$ and time $\times$ group effect, $\mathrm{F}$ $(12,616)=0.8, \mathrm{p}=0.614, \varepsilon=0.802)$. Introducing covariates in the analyses did not change these results.

\section{Associations between CAR and symptom development}

The percentage of patients with burnout (SMBQ $\geq 4$ ) decreased from $92 \%$ at inclusion to $64 \%$ at the 3-month

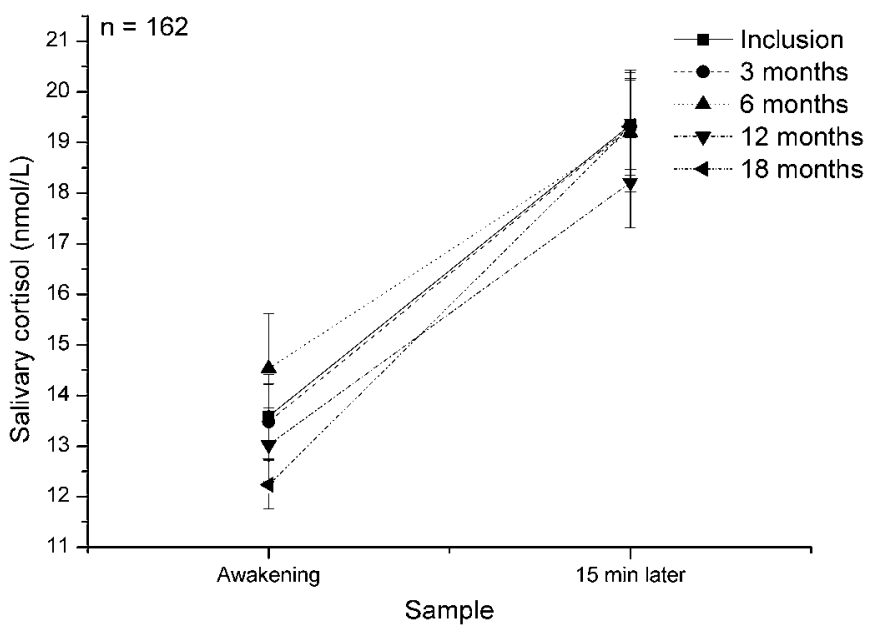

Figure 3 Mean cortisol awakening response ( \pm SEM) at inclusion and during the 18-month treatment programme. 
follow-up, $51 \%$ at 6 months, $41 \%$ at 12 months and, finally, 33\% at the 18-month follow-up. HAD-A scores $>10$ was seen in $60 \%$ of the patients at inclusion, $30 \%$ at 3 months, $20 \%$ at 6 months, $13 \%$ at 12 months and $9 \%$ at 18 months. The percentage of patients scoring HAD-D $>10$ decreased from $30 \%$ at inclusion to $12 \%$ at 3 months, $11 \%$ at 6 months, $8 \%$ at 12 months and $6 \%$ at 18 months. Further details regarding symptom development and clinical improvement during treatment have been presented elsewhere. ${ }^{25}$ No significant correlations were found between symptom development (SMBQ, HAD-D and HAD-A) during treatment and changes in CAR over the course of treatment (absolute $\rho=0.003-0.14$, $p$ values $=0.971-0.097)$.

\section{DISCUSSION}

The main findings in the present study were that patients with ED showed similar CAR to controls and that CAR did not change significantly during treatment.

\section{CAR in patients and healthy controls}

We confirm several previously published studies, showing no significant differences in awakening cortisol level and/or rise thereof between clinical populations of burnout subjects and controls ${ }^{13-15}$ but, as previously mentioned, contrasting results have been reported. ${ }^{11} 1231$ Thus, clinically diagnosed stress-related exhaustion does not seem to be related to changes in HPA axis activity, at least not to the CAR, as measured in this study.

A commonly suggested explanation for the heterogeneity in the literature concerning HPA axis in burnout is that HPA axis functioning changes as the burnout syndrome develops from enhanced work stress over heightened burnout scores to severe burnout or even clinical diagnosis of burnout. ${ }^{10} 1420$ It has been hypothesised that the earlier stages of burnout are characterised by increased levels of free cortisol, while the later stages are associated with lower than normal cortisol levels, representing a breakdown of endocrine feedback mechanisms. ${ }^{32} 33$ If these were the case, we would probably expect that clinically defined patients with exhaustion and high burnout scores would show altered CAR when compared with a control group. However, this was not found.

\section{CAR in subgroups of patients}

One possible explanation for the discrepancy between different studies, regarding HPA axis function, could be the presence of comorbidities. The clinical symptoms of other stress-related pathologies such as depression, posttraumatic stress disorder (PTSD), fibromyalgia and CFS partly overlap with symptoms seen in patients with burnout and exhaustion. These disorders show contrasting HPA axis disturbances, ${ }^{34}$ with higher cortisol levels as a characteristic of major depression, ${ }^{35} 36$ whereas PTSD, fibromyalgia and CFS are associated with hypofunction of the HPA axis. ${ }^{35-38}$ Thus, differences between cases and controls may, to a larger extent, actually be attributed to these comorbidities rather than solely to the presence of exhaustion and/or burnout. In this particular study, no patients with PTSD, CFS or fibromyalgia were included, but comorbid depression was present in the majority of the patients. The analysis with groupings of patients according to depression did not, however, reveal any differences in awakening cortisol between those who did and did not fulfil the diagnostic criteria for depression, indicating that comorbid depression does not contribute significantly in explaining the lack of difference between ED patients and controls.

A recent meta-analysis concluded that the relationship between HPA axis activity and depression is not as straightforward as often suggested. ${ }^{39}$ HPA axis hypoactivity has been related to atypical depression, which is also characterised by profound fatigue and inertia, whereas hypercortisolism is frequently observed in melancholic depression. ${ }^{40}$ A previous study of job stressinduced depression, with a population similar to the present ED population, showed a marked decrease of HPA axis reactivity to dexamethasone/corticotropinreleasing hormone challenge, which is opposite to what has been described in major depression. ${ }^{41}$ The lack of association between CAR and depressive symptoms, as observed in the present patient group and in the previous studies, ${ }^{14} 2031$ further complicates the picture. Impairments of psychological well-being are not necessarily related to easily detectable dysregulations of the HPA axis, and subjective stress reactions are not always reflected in cortisol responses. ${ }^{21}$

\section{Longitudinal development of CAR}

We found no significant changes in CAR over time in patients with ED despite clinical and psychological improvement during treatment. Similar results have been reported previously, ${ }^{22}{ }^{23}$ although a pilot study of 22 burnout patients reported increased awakening cortisol after psychotherapy. ${ }^{31}$ Moch et $a l^{22}$ suggested that the clinical improvement and reduction of symptoms may precede any accompanying change in biochemical markers. However, since our patient group did not differ from healthy controls at the time of inclusion, changes in CAR in any particular direction could perhaps not be expected.

Moreover, the lack of association between CAR and selfreported symptom duration before inclusion was somewhat puzzling, given the hypothesis above, and it also points in the direction that basal salivary cortisol is not a sensitive biological marker for exhaustion/burnout. We cannot, however, rule out that cortisol measured at other time points during the day, as in the evening, could be affected in this patient group, but the data on CAR does not implicate any substantial pathological change in the basal non-stimulated HPA activity. Further studies, measuring cortisol during other time points, might reveal whether the cortisol level is affected in this patient group.

\section{Methodological considerations}

Irrespective of the body of literature reporting no cortisol deviation in exhaustion/burnout populations, ${ }^{42}$ there still 
seems to be a strong belief that HPA axis dysregulation is a key aspect in the pathogenesis of stress-related illness. Our results do not rule out the possibility of a dysfunctional HPA axis in ED in terms of altered stress reactivity or altered feedback inhibition. Impaired habituation to repeated stress exposures or increased or decreased responses to acute stress are potential pathways by which stress-related illness may be linked to HPA axis dysfunction. Speculatively, any deviations in HPA axis activity could emerge when the stress system is challenged, as by a psychosocial stressor such as the Trier Social Stress Test or by dexamethasone suppression tests. Moreover, it is possible that a combination of several biomarkers, including HPA axis parameters, would be more sensitive in discriminating between ED or burnout cases and controls and thereby serve better as marker for stress-related illness.

Cortisol sampling points at awakening and $15 \mathrm{~min}$ later were chosen to ensure that salivary cortisol would be firmly on the upward slope of the morning cortisol response. $^{43}$ Moreover, memory deficits and cognitive impairments are a common feature in $\mathrm{ED},{ }^{41} 44$ and a shorter time span between sampling points was therefore desirable to facilitate remembering to perform the next sample, which would thereby increase compliance with sampling instructions. Collecting only two samples was, however, a limitation to the study since three or more samples are recommended to fully cover the awakening response. ${ }^{9}$ Resources for the current study did not allow a more extensive cortisol sampling protocol, given the extensive follow-up measurements.

The patient group included in the analyses differs somewhat compared with the patients who did not have complete samples of saliva during the follow-up. However, CAR at the time of inclusion did not differ between the 162 patients included in the study and the 112 excluded due to incomplete follow-up assessments $(\mathrm{t}=-0.2, \mathrm{p}=0.808)$. Hence, we do not think that such differences, including burnout level, were important.

To obtain a homogenous population of patients regarding stress-related exhaustion, we ensured that all patients fulfilled the diagnostic criteria for ED. Most studies on stress-related illness have instead used the concept of burnout. However, burnout is a psychological term with different definitions depending on the instrument used and with no clearly defined diagnostic criteria. Moreover, burnout has its focus on work stress and does not consider that stress-related illness can be due to stressors outside the workplace. Therefore, instead of using the burnout concept, we chose to only include patients with ED. Care was taken to ensure that no other somatic or psychiatric illness could explain the condition and special attention was given to exclude patients with fibromyalgia and CFS, since there is a considerable overlap between these conditions and ED. Patients with comorbid depression and/or anxiety were, however, not excluded from the study. Comorbid depression and anxiety is common in the patient group included in this study, and we thus confirm the previous literature that coexistence of depression, anxiety and burnout/exhaustion is often seen in patients reporting stress-related mental health problems. ${ }^{13} 45-47$ In a recent paper, published by our group, ${ }^{25}$ the course of symptoms for depression, anxiety and burnout was followed over 18 months, showing that symptoms indicating probable depression or anxiety declined more rapidly than symptoms of burnout. Thus, the core symptoms of these patients seem to be burnout, including physical and mental exhaustion, rather than depression and anxiety, but the overlap of these symptoms needs to be further studied. Some patients were on antidepressants, which potentially could influence HPA axis activity. However, the subgroup analyses in the patient group did not show any significant effects from antidepressant use.

The majority of the patients referred to the clinic report relatively high socioeconomic status, including high educational level, with few reporting unemployment. This should be acknowledged as a selection bias compared with the general population of patients with stress-related problems. Furthermore, patients seeking care for stress-related problems often report several other symptoms including musculoskeletal pain. The group of patients studied here differs somewhat from the general patient population in primary care mainly because patients with chronic pain problems are excluded since we are interested in studying patients primarily reporting stress-related exhaustion and burnout. The previous research on similar patient groups, showing changes in cortisol levels, could thus have been performed on different patient groups, such as those reporting more chronic pain problems.

\section{CONCLUSIONS}

CAR, as measured in the present study, does not seem to discriminate patients with clinically defined stress-related exhaustion from healthy controls, and CAR also appears not to change over the course of treatment. Thus, morning cortisol measurements in saliva do not seem to be a valid biological marker for exhaustion. The results do not refute a role for other aspects of the HPA axis in the long-term effects of stress since a limited measure of the HPA axis function was included in this study.

Acknowledgements We gratefully acknowledge Karin Nygren and MarieLouise Norberg for their valuable help in salivary cortisol analyses.

Contributors AS analyzed and interpreted the data and drafted the article, TL and IHJ contributed to conception and design of the study, interpretation of the data and revised the article for critical intellectual content. All authors approved the final version of the article.

Funding This research received no specific grant from any funding agency in the public, commercial or not-for-profit sectors.

Competing interests None.

Ethics approval The Regional Ethical Review Board in Gothenburg.

Provenance and peer review Not commissioned; externally peer reviewed.

Data sharing statement No additional unpublished data from the study are available. 


\section{REFERENCES}

1. Bültmann U, Huibers MJH, Van Amelsvoort LPGM, et al. Psychological distress, fatigue and long-term sickness absence: prospective results from the Maastricht Cohort Study. J Occup Environ Med 2005;47:941-7.

2. Maslach C, Schaufeli WB, Leiter MP, et al. Job burnout. Annu Rev Psychol 2001;52:397-422.

3. Grossi G, Perski A, Evengård B, et al. Physiological correlates of burnout among women. J Psychosom Res 2003;55:309-16.

4. National Board of Health and Welfare. Exhaustion Disorder [Utmattningssyndrom-Stressrelaterad Psykisk Ohälsa]. Stockholm: Socialstyrelsen, 2003.

5. Jonsdottir IH, Hägg DA, Glise K, et al. Monocyte chemotactic protein1 (MCP-1) and growth factors called into question as markers of prolonged psychosocial stress. PLOS ONE 2009;4:e7659.

6. Chrousos GP, Gold PW. The concepts of stress and stress system disorders: overview of physical and behavioral homeostasis. JAMA 1992;267:1244-52

7. Miller GE, Chen E, Zhou ES. If it Goes up, must it come down? Chronic stress and the hypothalamic-pituitary-adrenocortical axis in humans. Psychol Bull 2007;133:25-45.

8. Raison $\mathrm{CL}$, Miller $\mathrm{AH}$. When not enough is too much: the role of insufficient glucocorticoid signaling in the pathophysiology of stressrelated disorders. Am J Psychiatry 2003;160:1554-65.

9. Chida $\mathrm{Y}$, Steptoe A. Cortisol awakening response and psychosocia factors: a systematic review and meta-analysis. Biol Psychol 2009;80:265-78.

10. Kudielka BM, Bellingrath S, Hellhammer DH. Cortisol in burnout and vital exhaustion: an overview. G Ital Med Lav Ergon 2006;28 (Suppl 1):34-42.

11. De Vente W, Olff M, Van Amsterdam JGC, et al. Physiological differences between burnout patients and healthy controls: blood pressure, heart rate, and cortisol responses. Occup Environ Med 2003;60(Suppl 1):i54-i61.

12. Grossi G, Perski A, Ekstedt M, et al. The morning salivary cortisol response in burnout. J Psychosom Res 2005;59:103-11.

13. Österberg K, Karlson B, Hansen AM. Cognitive performance in patients with burnout, in relation to diurnal salivary cortisol. Stress 2009;12:70-81.

14. Mommersteeg PMC, Heijnen CJ, Verbraak MJPM, et al. Clinical burnout is not reflected in the cortisol awakening response, the day-curve or the response to a low-dose dexamethasone suppression test. Psychoneuroendocrinology 2006;31:216-25.

15. Mommersteeg PMC, Heijnen CJ, Kavelaars A, et al. Immune and endocrine function in burnout syndrome. Psychosom Med 2006;68:879-86.

16. Bellingrath S, Weigl T, Kudielka BM. Cortisol dysregulation in school teachers in relation to burnout, vital exhaustion, and effort-rewardimbalance. Biol Psychol 2008;78:104-13.

17. Pruessner JC, Hellhammer DH, Kirschbaum C. Burnout, perceived stress, and cortisol responses to awakening. Psychosom Med 1999;61:197-204.

18. Langelaan S, Bakker AB, Schaufeli WB, et al. Do burned-out and work-engaged employees differ in the functioning of the hypothalamic-pituitary-adrenal axis? Scand J Work Environ Health 2006;32:339-48.

19. Melamed S, Ugarten U, Shirom A, et al. Chronic burnout, somatic arousal and elevated salivary cortisol levels. J Psychosom Res 1999;46:591-8

20. Wingenfeld K, Schulz M, Damkroeger A, et al. Elevated diurnal salivary cortisol in nurses is associated with burnout but not with vital exhaustion. Psychoneuroendocrinology 2009;34:1144-51.

21. Sonnentag S. Burnout and functioning of the hypothalamus-pituitaryadrenal axis - there are no simple answer. Scand $J$ Work Environ Health 2006;32:333-7.

22. Moch S, Panz V, Joffe B, et al. Longitudinal changes in pituitaryadrenal hormones in South African women with burnout. Endocrine 2003;21:267-72.

23. Mommersteeg PMC, Heijnen CJ, Verbraak MJPM, et al. A longitudinal study on cortisol and complaint reduction in burnout. Psychoneuroendocrinology 2006;31:793-804.
24. Spitzer RL, Williams JBW, Kroenke K, et al. Utility of a new procedure for diagnosing mental disorders in primary care. JAMA 1994;272:1749-56

25. Glise K, Ahlborg G, Jonsdottir I. Course of mental symptoms in patients with stress-related exhaustion: does sex or age make a difference? BMC Psychiatry 2012;12:18.

26. Kirschbaum C, Kudielka BM, Gaab J, et al. Impact of gender, menstrual cycle phase, and oral contraceptives on the activity of the hypothalamus-pituitary-adrenal axis. Psychosom Med 1999;61:154-62.

27. Melamed S, Kushnir T, Shirom A. Burnout and risk factors for cardiovascular diseases. Behav Med 1992;18:53-60.

28. Stenlund T, Ahlgren C, Lindahl B, et al. Patients with burnout in relation to gender and a general population. Scand $J$ Public Health 2007;35:516-23.

29. Zigmond AS, Snaith RP. The hospital anxiety and depression scale. Acta Psychiat Scand 1983;67:361-70.

30. Bjelland I, Dahl AA, Haug TT, et al. The validity of the hospital anxiety and depression scale. An updated literature review. J Psychosom Res 2002;52:69-77.

31. Mommersteeg PMC, Heijnen CJ, Keijsers GPJ, et al. Cortisol deviations in people with burnout before and after psychotherapy: a pilot study. Health Psychol 2006;25:243-8.

32. McEwen BS. Stress, adaptation, and disease: allostasis and allostatic load. Ann N Y Acad Sci 1998;840:33-44.

33. Heim $\mathrm{C}$, Ehlert $\mathrm{U}$, Hellhammer $\mathrm{DH}$. The potential role of hypocortisolism in the pathophysiology of stress-related bodily disorders. Psychoneuroendocrinology 2000;25:1-35

34. Ehlert U, Gaab J, Heinrichs M. Psychoneuroendocrinological contributions to the etiology of depression, posttraumatic stress disorder, and stress-related bodily disorders: the role of the hypothalamus-pituitary-adrenal axis. Biol Psychol 2001;57:141-52.

35. Handwerger K. Differential Patterns of HPA activity and reactivity in Adult posttraumatic stress disorder and major depressive disorder. Harv Rev Psychiatry 2009;17:184-205.

36. Wingenfeld $\mathrm{K}$, Wolf OT. HPA axis alterations in mental disordersi impact on memory and its relevance for therapeutic interventions. CNS Neurosci Ther 2011;17:714-22.

37. Riva $\mathrm{R}$, Mork $\mathrm{P}$, Westgaard $\mathrm{R}$, et al. Fibromyalgia syndrome is associated with hypocortisolism. Int J Behav Med 2010;17:223-33.

38. Tak LM, Cleare AJ, Ormel J, et al. Meta-analysis and metaregression of hypothalamic-pituitary-adrenal axis activity in functional somatic disorders. Biol Psychol 2011;87:183-94.

39. Knorr U, Vinberg M, Kessing LV, et al. Salivary cortisol in depressed patients versus control persons: a systematic review and metaanalysis. Psychoneuroendocrinology 2010;35:1275-86.

40. Gold PW, Chrousos GP. Organization of the stress system and its dysregulation in melancholic and atypical depression: high vs low $\mathrm{CRH} / \mathrm{NE}$ states. Mol Psychiatry 2002;7:254

41. Rydmark I, Wahlberg K, Ghatan $\mathrm{PH}$, et al. Neuroendocrine, cognitive and structural imaging characteristics of women on longterm sickleave with job stress-induced depression. Biol Psychiatry 2006;60:867-73.

42. Danhof-Pont MB, van Veen T, Zitman FG. Biomarkers in burnout: a systematic review. J Psychosom Res 2011;70:505-24.

43. Bengtsson I, Lissner L, Ljung $\mathrm{T}$, et al. The cortisol awakening response and the metabolic syndrome in a population-based sample of middle-aged men and women. Metabolism 2010;59:1012-19.

44. Sandström A, Rhodin IN, Lundberg M, et al. Impaired cognitive performance in patients with chronic burnout syndrome. Biol Psychol 2005;69:271-9.

45. Sandström A, Peterson J, Sandström E, et al. Cognitive deficits in relation to personality type and hypothalamic-pituitary-adrenal (HPA) axis dysfunction in women with stress-related exhaustion. Scand $J$ of Psychol 2011;52:71-82.

46. Johannsen A, Rylander G, Söder B, et al. Dental plaque, gingival inflammation, and elevated levels of interleukin- 6 and cortisol in gingival crevicular fluid from women with stress-related depression and exhaustion. J Periodontol 2006;77:1403-9.

47. Sonnenschein M, Mommersteeg PMC, Houtveen $\mathrm{JH}$, et al. Exhaustion and endocrine functioning in clinical burnout: an in-depth study using the experience sampling method. Biol Psychol 2007;75:176-84. 